\title{
TT-FUNCTIONALS AND MARTIN-LÖF RANDOMNESS FOR BERNOULLI MEASURES
}

\author{
LOGAN AXON
}

\begin{abstract}
For $r \in[0,1]$, the Bernoulli measure $\mu_{r}$ on the Cantor space $\{0,1\}^{\mathbb{N}}$ assigns measure $r$ to the set of sequences with 1 at a fixed position. In [5] it is shown that for $r, s \in[0,1], \mu_{s}$ is continuously reducible to $\mu_{r}$ if and only if $r$ and $s$ satisfy certain purely number theoretic conditions (binomial reducibility). We bring these results into the context of computability theory and Martin-Löf randomness and show that the continuous maps arising in [5] are truthtable functionals (tt-functionals) on $\{0,1\}^{\mathbb{N}}$. This allows us to extend the characterization of continuous reductions between Bernoulli measures to include tt-functionals. It then follows from the conservation of randomness under tt-functionals that if $s$ is binomially reducible to $r$, then there is a tt-functional that maps every Martin-Löf random sequence for $\mu_{s}$ to a Martin-Löf random sequences for $\mu_{r}$. We are also able to show using results in 2 that the converse of this statement is not true.
\end{abstract}

\section{INTRODUCTION}

A functional $F:\{0,1\}^{\mathbb{N}} \rightarrow\{0,1\}^{\mathbb{N}}$ is a truth-table functional (ttfunctional) if there is a Turing machine $T$ with the property that $F(g)=h$ if and only if $T^{g}(i)=h(i)$ for all $i \in \mathbb{N}$ (where $T^{g}$ is the Turing machine $T$ equipped with oracle $g$ ). Such functionals give truth table reductions between infinite binary sequences. In this paper we investigate the interactions of tt-functionals with Martin-Löf random binary sequences.

Martin-Löf randomness combines probability theory with computability theory in a way that allows for the study of specific "random" binary sequences. The space $\{0,1\}^{\mathbb{N}}$ is usually equipped with the fair-coin measure: the measure which assigns a probability of $\frac{1}{2}$ to the occurrence of 1 in each position of the sequence. We will call this measure $\mu_{\frac{1}{2}}$. Truth table reductions and their interaction with Martin-Löf random binary sequences under the measure $\mu_{\frac{1}{2}}$ have been studied before, notably in [4]. More recently [2] considered truth table reductions of binary sequences that are Martin-Löf random under computable measures. We will consider truth table reductions of binary sequences that are Martin-Löf random under 


\section{TT-FUNCTIONALS FOR BERNOULLI MEASURES}

Bernoulli measures. When the Bernoulli measures are computable this is a special case of the situation studied in [2]. Our main result follows directly from the work of $[5]$ on continuous images of measures on $\{0,1\}^{\mathbb{N}}$ (Theorem 3.4 below). The observation that Theorem 3.4 is entirely effective together with Lemma 2.2 (recently given the name "Conservation of Randomness" in [2]) allows us to prove Theorem 3.6. We also note that results in [2] suffice to prove that the converse of Theorem 3.6 is not true.

Measures require sigma algebras and in this case we work with the Borel sigma algebra arising from the topology with a basis of cylinders: for a finite binary string $\sigma$, define the basic open cylinder $[\sigma]$ by

$$
[\sigma]=\left\{f \in\{0,1\}^{\mathbb{N}}: f \text { extends } \sigma\right\} .
$$

Under this topology $\{0,1\}^{\mathbb{N}}$ is compact, Hausdorff, and second countable (and homeomorphic to the Cantor middle thirds set, hence the name "Cantor space"). A subset $E \subseteq\{0,1\}^{\mathbb{N}}$ is clopen if both the subset $E$ and its complement $E^{\complement}$ are open. It follows from the compactness of the space $\{0,1\}^{\mathbb{N}}$, that the clopen subsets of $\{0,1\}^{\mathbb{N}}$ are exactly the finite unions of basic open cylinders. It is also worth noting that $E$ is clopen if and only if $E$ is $\Delta_{1}^{0}$ (that is, both $E$ and $E^{\complement}$ are $\Sigma_{1}^{0}$ ).

We also note that there are some issues related to the computational power of the measure under consideration. The definitions of Martin-Löf randomness include an oracle with sufficient computational power to calculate the measure of any basic open cylinder. The main complication is that there is no canonical choice of oracle, indeed as shown in [3] there may not be a minimal Turing degree with sufficient computational power. We are interested only in Bernoulli measures, however, which greatly simplifies the situation. In this case the Bernoulli parameter is the appropriate minimal oracle (a proof can be found in 1). We will initially state results and definitions for computable measures (that is, those for which the measure of any basic open cylinder is uniformly computable). These definitions and results may later be relativized to an appropriate oracle when dealing with non-computable measures.

We now recall the definition of Martin-Löf randomness.

Definition 1.1. Let $\mu$ be a computable Borel measure on $\{0,1\}^{\mathbb{N}}$, let $\sigma_{1}, \sigma_{2}$, $\sigma_{3}, \ldots$ be the standard enumeration of $\left.\{0,1\}\right\}^{<\mathbb{N}}$, and let $\langle\cdot, \cdot\rangle: \mathbb{N} \times \mathbb{N} \rightarrow \mathbb{N}$ be the standard computable pairing function.

(1) A sequence of subsets of $\{0,1\}^{\mathbb{N}},\left\{U_{i}\right\}_{i \in \mathbb{N}}$, is uniformly $\Sigma_{1}^{0}$ if there is a computable $f \in\{0,1\}^{\mathbb{N}}$ such that for each $i \in \mathbb{N}$

$$
U_{i}=\bigcup_{f(\langle i, n\rangle)=1}\left[\sigma_{n}\right]
$$

MISSOURI J. OF MATH. SCI., FALL 2015 


\section{AXON}

(2) A $\mu$-Martin-Löf test is a uniformly $\Sigma_{1}^{0}$ sequence of sets $\left\{U_{i}\right\}_{i \in \mathbb{N}}$ such that for each $i \in \mathbb{N}, \mu\left(U_{i}\right) \leq 2^{-i}$.

(3) A sequence $g \in\{0,1\}^{\mathbb{N}}$ is $\mu$-Martin-Löf random if there is no $\mu$ Martin-Löf test $\left\{U_{i}\right\}_{i \in \mathbb{N}}$ such that $g \in \bigcap_{i \in \mathbb{N}} U_{i}$.

We follow the custom of writing "Martin-Löf random" instead of " $\mu_{\frac{1}{2}}$ Martin-Löf random".

\section{Martin-Löf RAndomness And TT-Functionals}

We begin with an example. Define a functional $F:\{0,1\}^{\mathbb{N}} \rightarrow\{0,1\}^{\mathbb{N}}$ by $[F(g)](i)=0$ for all $i \in \mathbb{N}$. Then $F$ is a tt-functional that maps all sequences to the sequence consisting entirely of zeros, a sequence which is not Martin-Löf random. Hence, $F$ has the property of mapping Martin-Löf random sequences to a non-Martin-Löf random sequence.

We note that any tt-functional $F:\{0,1\}^{\mathbb{N}} \rightarrow\{0,1\}^{\mathbb{N}}$ is continuous and hence also gives a function between Borel measures on $\{0,1\}^{\mathbb{N}}$ (defined by $\mu \mapsto \mu \circ F^{-1}$ ). It turns out that this phenomenon of Martin-Löf random sequences mapping to non-Martin-Löf random sequences via a tt-functional only occurs when we fail to transform measures as well. This result is not new, but it may not be widely known, so we provide a statement and proof of the result in Lemma 2.2. This lemma has recently been given the name "Conservation of Randomness" in 2 .

Our proof of Lemma 2.2 relies on the following lemma.

Lemma 2.1. If $F:\{0,1\}^{\mathbb{N}} \rightarrow\{0,1\}^{\mathbb{N}}$ is a tt-functional, then $F^{-1}([\sigma])$ is a clopen set uniformly computable from $\sigma \in\{0,1\}<\mathbb{N}$.

Proof. Let $\sigma \in\{0,1\}<\mathbb{N}$.

We first prove that $F^{-1}([\sigma])$ is open. If $F(g) \in[\sigma]$, then by the finite use principle there is some $n \in \mathbb{N}$ such that if $h \in\{0,1\}^{\mathbb{N}}$ agrees with $g$ up to bit $n$, then $F(h) \in[\sigma]$. Consequently, if $\tau$ is the length $n$ initial segment of $g$, then $[\tau] \subseteq F^{-1}([\sigma])$. Therefore, $F^{-1}([\sigma])$ is open.

Because the cylinders form a basis for the topology on $\{0,1\}^{\mathbb{N}}$, it follows that $F$ is continuous. The cylinder $[\sigma]$ is also closed and hence, $F^{-1}([\sigma])$ must be closed. Therefore, $F^{-1}([\sigma])$ is clopen.

Let $T$ be a Turing machine associated with the functional $F$ (so that $\left.F(g)=T^{g}\right)$. For $\tau \in\{0,1\}^{<\mathbb{N}}$ let $T^{\tau}$ be the string given by $T^{\tau}(i)\lceil|\tau|$ for as many bits as this computation converges. Cantor space is compact and hence there is a smallest positive integer $n$ such that $T^{\tau}$ has at least length $|\sigma|$ for all $\tau \in 2^{n}$ (and $n$ may be computed uniformly from $\sigma$ ). Consequently, for $\tau \in 2^{n},[\tau] \subseteq F^{-1}([\sigma])$ if and only if $T^{\tau}$ is an extension of $\sigma$. Therefore, $F^{-1}([\sigma])$ is uniformly computable from $\sigma$. 


\section{TT-FUNCTIONALS FOR BERNOULLI MEASURES}

Lemma 2.2 (Conservation of Randomness). Let $F:\{0,1\}^{\mathbb{N}} \rightarrow\{0,1\}^{\mathbb{N}}$ be a tt-functional and let $\mu$ be a computable Borel measure on $\{0,1\}^{\mathbb{N}}$. If $g \in$ $\{0,1\}^{\mathbb{N}}$ is Martin-Löf random under the measure $\mu$, then $F(g)$ is Martin-Löf random under the measure $\mu \circ F^{-1}$.

Proof. Let $\left\{U_{i}\right\}_{i \in \mathbb{N}}$ be a $\left(\mu \circ F^{-1}\right)$-Martin-Löf test and let $h \in \bigcap_{i \in \mathbb{N}} U_{i}$. It follows from Lemma 2.1 that $\left\{F^{-1}\left(U_{i}\right)\right\}_{i \in \mathbb{N}}$ is a uniformly $\Sigma_{1}^{0}$ sequence of sets. Clearly $\mu\left(F^{-1}\left(U_{i}\right)\right)=\left(\mu \circ F^{-1}\right)\left(U_{i}\right) \leq 2^{-i}$. Therefore, $\left\{F^{-1}\left(U_{i}\right)\right\}_{i \in \mathbb{N}}$ is a $\mu$-Martin-Löf test. Clearly $F^{-1}(\{h\}) \subseteq \bigcap_{i \in \mathbb{N}} F^{-1}\left(U_{i}\right)$. Consequently if $g \in F^{-1}(\{h\})$, then $g$ is not $\mu$-Martin-Löf random. We have shown that if $F(g)=h$ and $h$ is not Martin-Löf random under $\mu \circ F^{-1}$, then $g$ is not Martin-Löf random under $\mu$. This is the contrapositive of the desired statement.

Note that our example of a functional that maps all sequences to the sequence of zeros shows that the converse of this theorem is not generally true (in this example the sequence of zeros is $\left(\mu_{\frac{1}{2}} \circ F^{-1}\right)$-Martin-Löf random, but also the image of non-Martin-Löf random sequences).

\section{Bernoulli Measures and TT-Functionals}

The fair coin measure arises naturally when considering a sequence of flips of a fair coin (hence the name). A natural generalization is to flip a biased coin, in which case the probability of heads or tails on each flip remains constant, but is no longer $\frac{1}{2}$. Such measures are known as Bernoulli measures because they arise from repeated, independent Bernoulli trials (such as flips of a coin).

Definition 3.1. Let $r \in[0,1]$. Define $\mu_{r}$ to be the Bernoulli measure on $\{0,1\}^{\mathbb{N}}$ given by $\mu_{r}\left(\left\{f \in\{0,1\}^{\mathbb{N}}: f(i)=1\right\}\right)=r$ for any $i \in \mathbb{N}$.

In light of the result of the last section, we might wonder when there is a tt-functional that maps the set of Martin-Löf random sequences for a particular Bernoulli measure into the set of Martin-Löf randoms sequences for another. Most of the work required to answer this question has already been done in [5], from which much of the following is derived.

Definition 3.2. Let $\mu$ and $\nu$ be Borel measures on Cantor Space. The measure $\nu$ is continuously reducible to measure $\mu$ if there is a continuous functional $F:\{0,1\}^{\mathbb{N}} \rightarrow\{0,1\}^{\mathbb{N}}$ such that $\nu=\mu \circ F^{-1}$ (meaning that $\nu(E)=\mu\left(F^{-1}(E)\right)$ for every Borel subset $E$ ). If $F$ is a tt-functional, then $\nu$ is computably reducible to $\mu$.

MISSOURI J. OF MATH. SCI., FALL 2015 


\section{AXON}

Definition 3.3. A partition polynomial is any polynomial $P(x)$ that can be expressed in the form

$$
P(x)=\sum_{i=0}^{n} a_{i} x^{i}(1-x)^{n-i}
$$

where $n$ is the degree of $P(x)$ and $a_{i}$ is an integer with $0 \leq a_{i} \leq\left(\begin{array}{c}n \\ i\end{array}\right)$ for $i=0,1, \ldots, n$. Let $r, s \in[0,1]$. Then $s$ is binomially reducible to $r$ if there is a partition polynomial $P(x)$ such that $s=P(r)$.

Theorem 3.4 (Mauldin). The measure $\mu_{s}$ is continuously reducible to $\mu_{r}$ if and only if $s$ is binomially reducible to $r$.

We provide the proof of Theorem 3.4 given in [5] in order to extend its application to tt-functionals and prove Corollary 3.5

Proof. We first prove that if $\mu_{s}$ is continuously reducible to $\mu_{r}$, then $s$ is binomially reducible to $r$. Suppose $F:\{0,1\}^{\mathbb{N}} \rightarrow\{0,1\}^{\mathbb{N}}$ is continuous and for each Borel set $E \subset\{0,1\}^{\mathbb{N}} \mu_{s}(E)=\mu_{r}\left(F^{-1}(E)\right)$. Consider the clopen set $[1] \subseteq\{0,1\}^{\mathbb{N}}$. By definition $s=\mu_{s}([1])=\mu_{r}\left(F^{-1}([1])\right)$. The functional $F$ is continuous and hence, $F^{-1}([1])$ is a clopen subset of $\{0,1\}^{\mathbb{N}}$. It follows that there is a positive integer $n$ and a collection $\mathcal{E} \subseteq 2^{n}$ such that

$$
F^{-1}([1])=\bigcup_{\sigma \in \mathcal{E}}[\sigma] .
$$

Let $a_{i}$ be the number of strings in $\mathcal{E}$ with exactly $i$ ones (and $n-i$ zeros). Thus, $0 \leq a_{i} \leq\left(\begin{array}{c}n \\ i\end{array}\right)$ for $i=0,1, \ldots, n$ and

$$
s=\mu_{r}\left(F^{-1}([1])\right)=\sum_{i=0}^{n} a_{i} r^{i}(1-r)^{n-i} .
$$

This is a binomial reduction of $s$ to $r$.

Conversely, suppose that there is a partition polynomial $P(x)=\sum_{i=0}^{n}$ $a_{i} x^{i}(1-x)^{n-i}$ such that $s=P(r)$. Let $\mathcal{E}$ be a subset of $2^{n}$ (where $n$ is the degree of the partition polynomial $P$ ) with exactly $a_{i}$ members having $i$ ones (and $n-i$ zeros). If $g \in\{0,1\}^{\mathbb{N}}$, then $g$ has a unique representation as a sequence of strings of length $n$ :

$$
g=\tau_{1}^{\imath} \tau_{2}^{\imath} \tau_{3}^{\imath} \cdots
$$

where $\tau_{i} \in 2^{n}$ for every $i$. Define a functional $F:\{0,1\}^{\mathbb{N}} \rightarrow\{0,1\}^{\mathbb{N}}$ by

$$
[F(g)](i)= \begin{cases}1 & \text { if } \tau_{i} \in \mathcal{E} \\ 0 & \text { otherwise }\end{cases}
$$

It is easy to verify that $F$ is continuous. 


\section{TT-FUNCTIONALS FOR BERNOULLI MEASURES}

We must now prove that

$$
\mu_{s}\left(\left\{h \in\{0,1\}^{\mathbb{N}}: h(k)=1\right\}\right)=\mu_{r}\left(F^{-1}\left(\left\{h \in\{0,1\}^{\mathbb{N}}: h(k)=1\right\}\right)\right)
$$

for any non-negative integer $k$ (see Definition 3.1). Our definition of $F$ means that

$$
F^{-1}\left(\left\{h \in\{0,1\}^{\mathbb{N}}: h(k)=1\right\}\right)=\left\{g \in\{0,1\}^{\mathbb{N}}: \tau_{k} \in \mathcal{E}\right\}
$$

(where $\tau_{k}$ is the $k$ th length $n$ string in $g$ ). Consequently

$$
\mu_{r}\left(F^{-1}\left(\left\{h \in\{0,1\}^{\mathbb{N}}: h(k)=1\right\}\right)\right)=\mu_{r}\left(\left\{g \in\{0,1\}^{\mathbb{N}}: \tau_{k} \in \mathcal{E}\right\}\right)=P(r) .
$$

Our initial assumption was that $P(r)=s$. Moreover,

$$
\mu_{s}\left(\left\{h \in\{0,1\}^{\mathbb{N}}: h(k)=1\right\}\right)=s
$$

by definition. Thus we have completed the proof.

The proof we have just given for Theorem 3.4 is entirely constructive and so the following corollary follows immediately.

Corollary 3.5. The measure $\mu_{s}$ is computably reducible to measure $\mu_{r}$ if and only if $s$ is binomially reducible to $r$.

Proof. First suppose that $\mu_{s}$ is computably reducible to $\mu_{r}$. Be definition there is a tt-functional $F:\{0,1\}^{\mathbb{N}} \rightarrow\{0,1\}^{\mathbb{N}}$ such that $\mu_{s}=\mu_{r} \circ F^{-1}$. By Lemma $2.1 \mathrm{~F}$ is continuous and thus $\mu_{s}$ is continuously reducible to $\mu_{r}$. By the preceding theorem $s$ is binomially reducible to $r$.

Now suppose that $s$ is binomially reducible to $r$. The proof of Theorem 3.4 shows how to construct a functional $F:\{0,1\}^{\mathbb{N}} \rightarrow\{0,1\}^{\mathbb{N}}$ such that $\mu_{s}=\mu_{r} \circ F^{-1}$. Although originally intended only to produce a continuous functional, this construction actually produces a tt-functional. Therefore, $\mu_{s}$ is computably reducible to $\mu_{r}$.

Our main result now follows.

Theorem 3.6. If $s$ is binomially reducible to $r$, then there is a tt-functional that maps the set of $\mu_{s}$-Martin-Löf random sequences into the set of $\mu_{r}$ Martin-Löf random sequences.

Proof. If $s$ is binomially reducible to $r$, then by Corollary $3.5 \mu_{s}$ is computably reducible to measure $\mu_{r}$. Let $F$ be a tt-functional corresponding to this reduction. Then by Lemma2.2 (Conservation of Randomness) $F$ maps the set of $\mu_{s}$-Martin-Löf random sequences into the set of $\mu_{r}$-Martin-Löf random sequences.

MISSOURI J. OF MATH. SCI., FALL 2015 


\section{AXON}

We note that the converse is not true. In [2] it is shown that for any atomless computable measure $\nu$ there is a tt-functional $F$ such that any $g \in\{0,1\}^{\mathbb{N}}$ is $\left(\nu \circ F^{-1}\right)$-Martin-Löf random if and only if $g$ is MartinLöf random (which we recall means $\mu_{\frac{1}{2}}$-Martin-Löf random). Applying Conservation of Randomness (Lemma 2.2) to this functional $F$ shows that if $g \in\{0,1\}^{\mathbb{N}}$ is $\nu$-Martin-Löf random, then $F(g)$ is Martin-Löf random. Any Bernoulli measure $\mu_{r}$ is atomless and computable when $r$ is computable, so this result applies for such measures. In particular, applying it to $\mu_{\frac{1}{3}}$ shows that there is a tt-functional $F$ such that if $g$ is $\mu_{\frac{1}{3}}$-Martin-Löf random, then $F(g)$ is Martin-Löf random. We thus have a tt-functional $F$ that maps the set of $\mu_{\frac{1}{3}}$-Martin-Löf random sequences into the set of $\mu_{\frac{1}{2}}$-MartinLöf random sequences. It is fairly straightforward to show that $\frac{1}{3}$ is not binomially reducible to $\frac{1}{2}$. Therefore the converse of Corollary 3.6 does not hold.

\section{REFERENCES}

[1] L. M. Axon, Martin-Löf randomness in spaces of closed sets, Journal of Symbolic Logic, 80.2 (2015), 359-383.

[2] L. Bienvenu and C. Porter, Strong reductions in effective randomness, Theoretical Computer Science, 459 (2012), 55-68.

[3] A. R. Day and J. S. Miller, Randomness for non-computable measures, Transactions of the American Mathematical Society, 365 (2013), 3575-3591.

[4] O. Demuth, Remarks on the structure of tt-degrees based on constructive measure theory, Commentationes Mathematicae Universitatis Carolinae, 29.2 (1988), 233247.

[5] R. D. Mauldin, Open Problems in Topology, North-Holland, Amsterdam, 1990, 617629.

MSC2010: 03D32, 68Q30

Key words and phrases: Cantor space, Martin-Löf randomness, truth-table reduction, Bernoulli measure

Department of Mathematics, Gonzaga University, 502 E Boone Ave, Spokane, WA 99258

E-mail address: axon@gonzaga.edu 